\title{
Performance Analysis of a Ultra-Compact Low-Power Rectenna in Paper Substrate for RF Energy Harvesting
}

\author{
V. Palazzi ${ }^{1}$, C. Kalialakis ${ }^{2}$, F. Alimenti ${ }^{1}$, P. Mezzanotte ${ }^{1}$, L. Roselli ${ }^{1}$, A. Collado ${ }^{3}$, A. Georgiadis ${ }^{3}$ \\ ${ }^{1}$ Department of Engineering, University of Perugia, Perugia, Italy \\ ${ }^{2}$ Centre Tecnològic de Telecomunicacions de Catalunya (CTTC), Castelldefels, Spain \\ ${ }^{3}$ Heriot-Watt University, School of Engineering and Physical Sciences, Edinburgh EH14 4AS, UK
}

valentina.palazzi@studenti.unipg.it

\begin{abstract}
In this paper the experimental results of a compact low-power rectenna in paper substrate, designed to operate in the Wi-Fi band, are presented. The complete prototype, based on an annular slot antenna and a single-diode rectifier, features a weight of 1.5 grams and shows an RF-to-dc conversion efficiency in the design band of about $40 \%$ for a $\mathbf{- 1 0} \mathbf{~ d B m}$ available input power, of about $28 \%$ at $\mathbf{- 1 5} \mathrm{dBm}$, and in the range [10, 22] \% at $\mathbf{- 2 0} \mathrm{dBm}$, corresponding to an output DC voltage in the order of 320,240 and $60 \mathrm{mV}$ respectively. Additionally, the rectenna features an efficiency higher then $7 \%$ in the whole band 1.8-2.7 dBm for a power density estimated around $3 \mu \mathrm{W} / \mathrm{cm}^{2}$.

Index Terms-green electronics, rectennas, Internet of Things, circuits on paper, copper laminate technology, rectifiers, slot antennas, wireless power transfer, energy harvesting
\end{abstract}

\section{INTRODUCTION}

With the increasing number of distributed and ubiquitus electronic apparatuses in the environment the power supply is emerging as one of the main challenges to face. In fact, in order to free the user from the burden of the battery management (both in terms of the need for periodical recharging and waste disposal), a lot of research is focusing on the development of batteryless, autonomous devices and nodes [1].

As a consequence, wireless power transfer and energy scavenging techniques are becoming of paramount importance. More than ever, energy harvesters with good performance are being developed in order to harness energy from different sources. Even new materials and compact solutions are being investigated in order to fit the different environments and applications (wearable sensors, portable devices, and so forth) [2], [3].

The aim of this paper is, on the one hand, to experimentally validate the simulation results reported in [4] and, on the other hand, to further investigate the performance of a low-power RF energy harvester in paper substrate.

\section{RECTENNA OVERVIEW}

A photograph of the proposed harvester is reported in Fig. 1. The rectenna features a double layer structure: the bottom

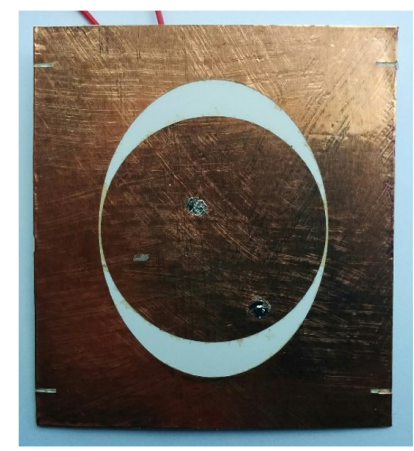

(a)

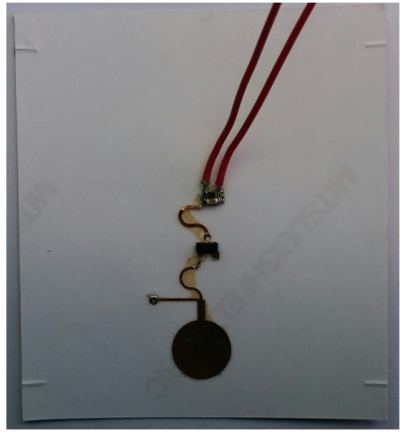

(b)
Fig. 1. Photograph of the rectenna prototype: antenna side (a) and circuitry side (b). The annular slot is the result of the intersection of a circle (radius = $15.8 \mathrm{~mm})$ and ellipse $\left(r_{1}=16.05 \mathrm{~mm}\right.$ and $\left.r_{2}=19.85 \mathrm{~mm}\right)$. Active area: $40 \mathrm{~mm}$ in length and $33 \mathrm{~mm}$ in width. Ground plane dimension: $49 \times 54 \mathrm{~mm}^{2}$.

side hosts the tapered annular slot antenna, fully characterized in [4], whereas the circuitry (including the proximity-coupled antenna feed line, the rectifier and the utilizer), implemented in microstrip technology, is placed on the top side. In particular, the rectifying circuit harnesses the interior metal surface of the annular slot as a ground plane, so that the active area of the proposed planar rectenna corresponds to the bare antenna area.

The schematic of the adopted rectifier is shown in Fig. 3. A series-connected single-diode rectifier has been chosen since this topology has been proven to be the most suitable for maximizing the RF-to-dc conversion efficiency in power scarce applications [5], [6]. The circuit is then completed by a distributed matching network and an RC filter. The lines have been folded in order to fit the rectifier layout in the antenna interior area. The design, aimed at maximizing the rectifying efficiency for available input powers in the range $[-20,-15] \mathrm{dBm}$ in the Wi-Fi band $(2.4-2.5 \mathrm{GHz})$, has been carried out within the ADS-Momentum suite. Both the line dimensions and the output load has been set as paramenters 
for the optimization. In the end, the whole rectenna consists of 3 lumped components, i. e., a low-barrier Schottky diode, a $100 \mathrm{pF}$ capacitor and a $2.7 \mathrm{k} \Omega$ resistor.

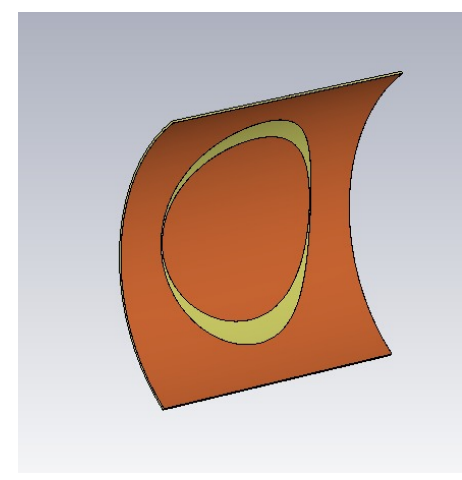

(a)

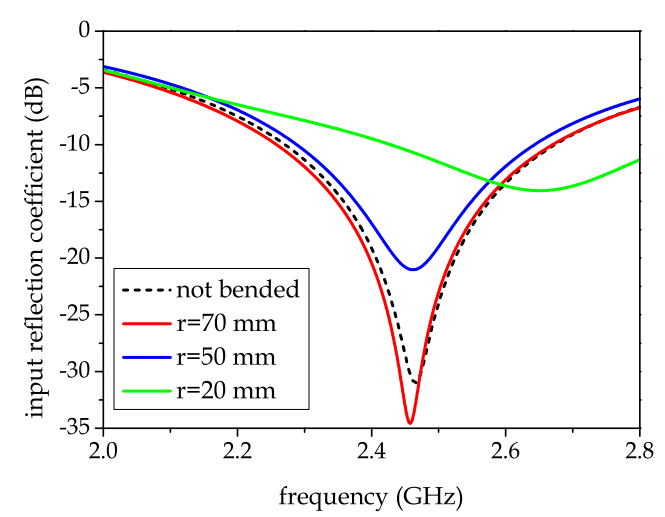

(b)

Fig. 2. Study of the impact of bending over the antenna performance: simulated structure (a) and input reflection coefficient for various radii of curvature (b)

The prototype is fabricated on a $230-\mu \mathrm{m}$ thick paper sheet from Mitsubishi $\left(\epsilon_{r}=2.55 \tan \delta=0.05\right)$ by means of the copper $(\mathrm{Cu})$ tape technology, which consists of the shaping of an adhesive copper tape by standard photolithography and the application of the $\mathrm{Cu}$ pattern on the hosting substrate by means of a sacrificial layer [7]. In the present case the top and the bottom side have been separately tackled and then aligned on the two sides of the same substrate. The final prototype features a weight of just 1.5 grams.

Since the rectenna is fabricated on a flexible substrate, it is susceptible to be bended (intentionally or not). To this purpose, a study of the impact of the bending over the antenna radiation performance has been carried out via electromagnetic simulation (see Fig. 2 (a)). It highlights that for a small bending (radius of curvature higher than $70 \mathrm{~mm}$ ) the antenna performance is substantially unchanged (see Fig. 2 (b)). On the other hand, although higher bending worsens the input matching of the antenna, the impact can be considered moderate and the WiFi band is still covered for a radius of curvature as small as $20 \mathrm{~mm}$. Moreover, the estimated reduction of the maximum antenna gain is lower than $1 \mathrm{~dB}$.

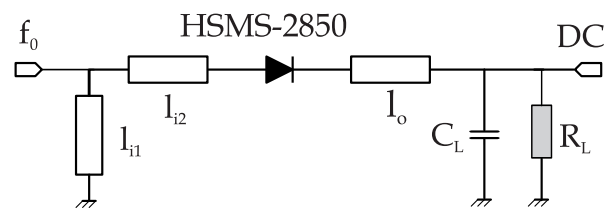

Fig. 3. Schematic of the developed rectifier. The main circuit parameters are: $l_{i 1}=6.7 \mathrm{~mm}, l_{i 2}=10.8 \mathrm{~mm}, w_{\text {stub }}=0.4 \mathrm{~mm}, w_{\text {line }}=0.28 \mathrm{~mm}$, $l_{o}=12.1 \mathrm{~mm}, w_{o}=0.4 \mathrm{~mm}$. The low-pass filter is a RC circuit with $C_{L}=100 \mathrm{pF}$ and $R_{L}=2.7 \mathrm{k} \Omega$.

\section{EXPERIMENTAL RESULTS}

In order to experimentally characterize the rectenna performance the following experiment has been set up (see Fig. 4). Both the presented rectenna prototype and the standalone tapered annular slot antenna have been aligned and placed in such a way to be at the same distance from the transmitting antenna (so as to receive approximately the same RF input power). The standalone antenna has been connected by using a standard SMA connector and a coaxial cable to a spectrum analyzer, whereas the rectenna has been connected by means of two wires to a digital multimeter. As a consequence, the actual available power at the input of the rectifier is extracted with reasonable accuracy, thus allowing an analysis of both the rectifier and the whole rectenna performance.

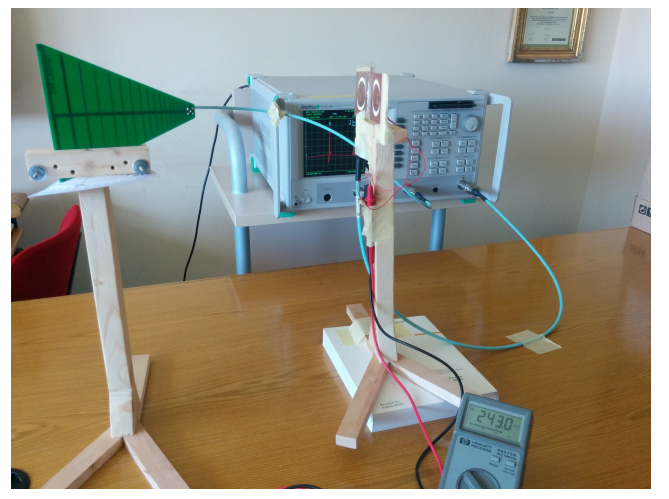

Fig. 4. Rectenna measurement: experimental setup. The generator is connected to a log-periodic antenna featuring a gain of $4 \mathrm{dBi}$ in the band 1.8-2.7 $\mathrm{GHz}$. The standalone slot antenna is connected to a spectrum analyzer, while the rectenna is connected to a multimeter.

Fig. 5 reports both the output $\mathrm{DC}$ voltage $\left(\mathrm{V}_{\mathrm{DC}}\right)$ and the RF-to-dc efficiency as a function of the available power at the input of the rectifying circuit $\left(\mathrm{P}_{\mathrm{avs}}\right)$ in the design band (2.4-2.5 $\mathrm{GHz}$ ), where the efficiency has been calculated by applying the equation (1):

$$
\eta_{R F-d c}=\frac{V_{D C}^{2} / R_{L}}{P_{a v s}} \times 100 .
$$

The experiment has been conducted by keeping a constant distance between the rectenna and the transmitting antenna (around $20 \mathrm{~cm}$ ) and varying the power transmitted by the source generator from -5 to $10 \mathrm{dBm}$. The measurement results agree very well with the simulations, with a slight improvement, which can be noticed at 2.4 and $2.45 \mathrm{GHz}$, and a slight 


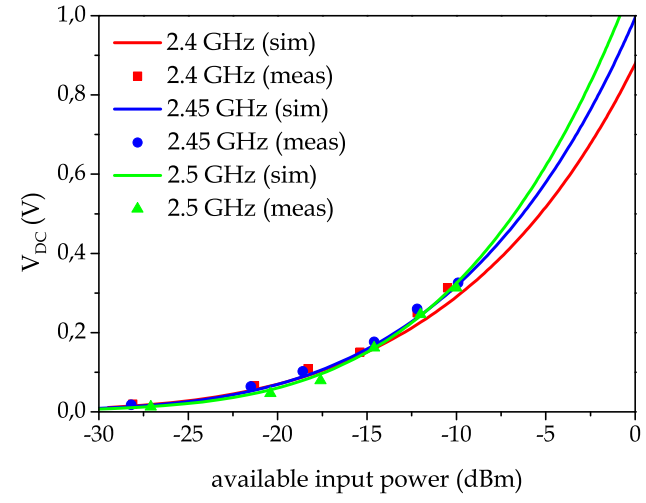

(a)

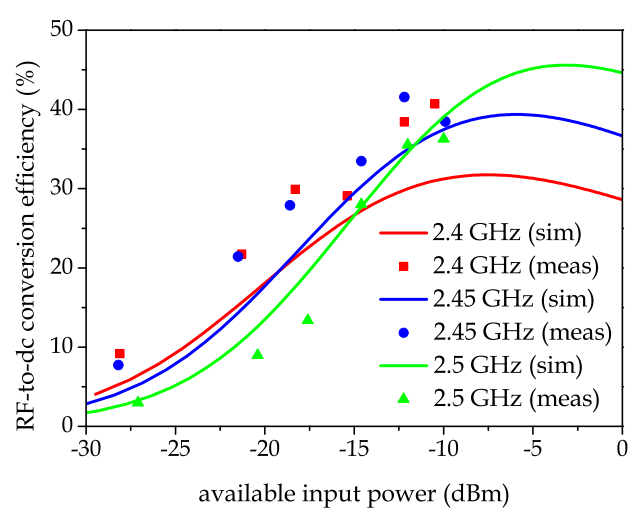

(b)

Fig. 5. Rectenna measurement: output DC voltage (a) and RF-to-DC efficiency (b) versus available power at the input of the rectifier in the band 2.4-2.5 GHz.

deterioration at $2.5 \mathrm{GHz}$. In particular, the rectenna features an efficiency of $40 \%$ around $-10 \mathrm{dBm}$, of $28 \%$ around $-15 \mathrm{dBm}$, and in the range $[10,22] \%$ around $-20 \mathrm{dBm}$, corresponding to an output DC voltage in the order of 320,240 and $60 \mathrm{mV}$ respectively.

Finally, in Fig. 6 the output DC voltage and efficiency are presented as a function of frequency, in order to check the rectenna performance even outside the design band. In this case the transmitted power has been kept fixed to $10 \mathrm{dBm}$ and the measurements have been recorded for two different transmitter-to-rectenna distances $(20$ and $30 \mathrm{~cm})$. It is worth noticing that also in this case the efficiency is calculated with the equation (1), by considering the measured RF input power captured by the standalone antenna. On the other hand, the power density $(\mathrm{S})$ is calculated dividing the measured RF input power by the effective area of the antenna, estimated with the well-known equation (2):

$$
A_{e f f}=G \frac{\lambda^{2}}{4 \pi}
$$

where $\mathrm{G}$ is the gain of the annular slot, and $\lambda$ is the wavelength. In particular, the power density has been calculated

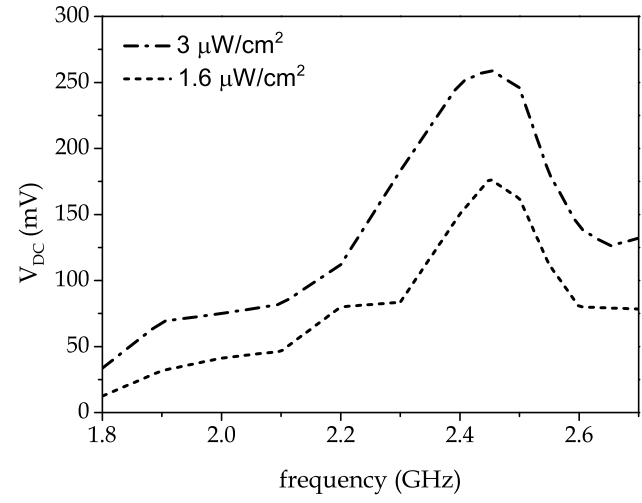

(a)

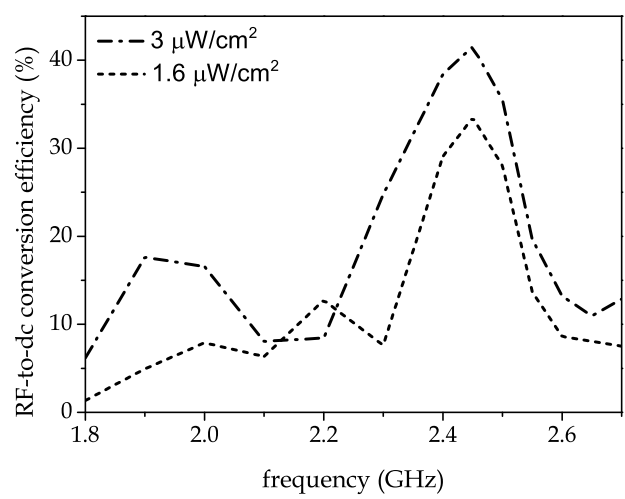

(b)

Fig. 6. Rectenna measurement: RF-to-dc conversion efficiency (a) and output voltage (b) as a function of frequency.

for five different frequencies $(1.9,2.1,2.4,2.5$ and $2.6 \mathrm{GHz})$ and the results have been averaged in order to compensate for uncertainties coming out of the experimental setup.

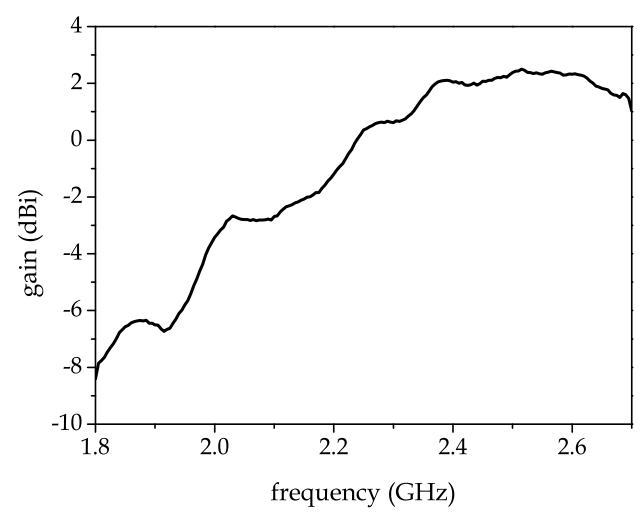

Fig. 7. Broadside gain of the tapered annular slot.

As it can be seen in Fig. 6, the rectenna is still able to harvest a useful voltage from both the UMTS 2100 band (1.922.17 GHz) and the new LTE band (2.5-2.69 GHz), although 
it operates outside its design band. In fact, state-of-the art low power sensors and DC/DC converters can nowadays be activated with voltages below $200 \mathrm{mV}$ [8] and up to $20 \mathrm{mV}$ [9]. This is mainly due to the tapered annular slot, whose gain decreases quite smoothly outside the band of interest (see Fig. 7), making this rectenna suitable for broadband and multi-band energy harvesting.

A rectenna based on a similar tapered annular slot antenna has also been reported in [10]. The latter rectenna works in the UHF mobile telephony bands (700-900 MHz), is based on a Dickson-charge pump and is fabricated on the TLY-5-0100 Taconic substrate, featuring a fractional bandwidth of $25 \%$.

\section{CONCLUSIONS}

A performance analysis of a low-power rectenna in paper substrate has been presented. Both a description of the rectenna prototype and the experimental setup have been reported. A good agreement between simulations and measurements have been noticed. In particular, the RF-to-dc conversion efficiency of $28 \%$ has been observed at $-15 \mathrm{dBm}$ of available input power, thus fully confirming the study reported in [4].

As future work, a study to enhance the antenna directivity will be performed to mitigate multipath effects, both by developing and testing an array of annular slots and by using a cavity-backed annular slot, so as to make the rectenna more robust and reliable.

\section{ACKNOWLEDGMENT}

The work was supported by the EU COST Action IC1301 WiPE (Wireless Power Transmission for sustainable Electronics). The work of Christos Kalialakis and Apostolos Georgiadis was also supported by the EU Horizon 2020 Research and Innovation Programme under the Marie Sklodowska-Curie Grant Agreements No 654734 and No 661621 respectively.

\section{REFERENCES}

[1] A. Costanzo, D. Masotti, "Smart Solutions in Smart Spaces: Getting the Most from Far-Field Wireless Power Transfer," in IEEE Microwave Magazine, vol.76, no.5, pp.30-45, May 2016.

[2] G. Monti, L. Corchia, L. Tarricone, "UHF wearable rectenna on textile materials," in IEEE Transactions on Antennas and Propagation, vol.61, no.7, pp.3869-3873, July 2013.

[3] S. Korhummel, D. G. Kuester, and Z. Popovic, "A HarmonicallyTerminated Two Gram Low-Power Rectenna on Flexible Substrate," in IEEE Wireless Power Transfer Conference (WPTC), pp. 119-122, 15-16 May 2013

[4] V. Palazzi, C. Kalialakis, F. Alimenti, P. Mezzanotte, L. Roselli, A. Collado, A. Georgiadis, "Design of a Ultra-Compact Low-Power Rectenna in Paper Substrate for Energy Harvesting in the Wi-Fi Band,' in IEEE Wireless Power Transfer Conference (WPTC), pp. 1-4, 5-6 May 2016.

[5] A. Boaventura, D. Belo, R. Fernandes, A. Collado, A. Georgiadis, and N. B. Carvalho, "Boosting the Efficiency: Unconventional Waveform Design for Efficient Wireless Power Transfer," in IEEE Microwave Magazine, vol.16, no.3, pp.87-96, April 2015.

[6] C. H. p. Lorenz, S. Hemour, and K. Wu, "Physical Mechanism and Theoretical Foundation of Ambient RF Power Harvesting Using Zero-Bias Diodes", in IEEE Transactions on Microwave Theory and Techniques, vol. 64, no. 7, pp. 2146-2158, July 2016.

[7] V. Palazzi, C. Mariotti, F. Alimenti, M. Virili, G. Orecchini, P. Mezzanotte, L. Roselli, "Demonstration of a Chipless Harmonic Tag working as Crack Sensor for Electronic Sealing Applications", in Wireless Power Transfer, 2, pp 78-85, October 2015. doi:10.1017/wpt.2015.11.
[8] S. Hanson, b. Zhai, M. Seok, B. Cline, K. Zhou, M. Singhal, M Minuth, J. Olson, L. Nazhandali, T. Austin, D. Sylvester, and D. Blaauw, "Exploring Variability and Performance in a Sub-200-mV Processor", in IEEE Journal of Solid-State Circuits, VOL. 43, N. 4, April 2008.

[9] Linear Technology, "TC3108 - Ultralow Voltage StepUp Converter and Power Manager," online website: http://www.linear.com/product/LTC3108

[10] V. Palazzi, M. Del Prete, M. Fantuzzi, "Scavenging for Energy: A Rectenna Design for Wireless Energy Harvesting in UHF Mobile Telephony Bands," in IEEE Microwave Magazine, to be published. 